\title{
Real-time monitoring of a mass K2-related overdose outbreak - Connecticut, 2018
}

Kristen Soto, Erin Grogan, Alexander Senetcky, Susan Logan

Epidemiology, Connecticut Department of Public Health, Hartford, Connecticut, United States

Objective

To describe the use of syndromic surveillance data for real-time situational awareness of emergency department utilization during a localized mass overdose event related to the substance K2.

\section{Introduction}

On August 15, 2018, the Connecticut Department of Public Health (DPH) became aware of a cluster of suspected overdoses in an urban park related to the synthetic cannabinoid K2. Abuse of K2 has been associated with serious adverse effects and overdose clusters have been reported in multiple states. This investigation aimed to characterize the use of syndromic surveillance data to monitor a cluster of suspected overdoses in real time.

\section{Methods}

The EpiCenter syndromic surveillance system collects data on all emergency department (ED) visits at Connecticut hospitals. ED visits associated with the event were identified using ad hoc keyword analyses. The number of visits by facility location for the state, county, and city were communicated to state and local partners in real time. Gender, age, and repeated ED visits were assessed. After the event, surveillance findings were summarized for partners

\section{Results}

During the period of August 15-16, 2018 the number of ED visits with a mention of K2 in the chief complaint increased from three to 30 in the impacted county, compared to a peak of 5 visits during the period of March-July, 2018. An additional 25 ED visits were identified using other related keywords (e.g., weed). After the event, 72 ED visits were identified with K2 and location keywords in the chief complaint or triage notes. These 72 visits comprised 53 unique patients, with 12 patients returning to the ED 2-5 times over the two day period. Of 53 patients, $77 \%$ were male and the median age was 40 years (interquartile range $35-51$ years). Surveillance findings were shared with partners in real time for situational awareness, and in a summary report on August 21.

\section{Conclusions}

Data from the EpiCenter system were consistent with reports from other data sources regarding this cluster of suspected drug overdoses. Next steps related to this event involve: monitoring data for reference to areas of concentrated substance use, enabling automated alerts to detect clusters of interest, and developing a plan to improve coordinate real-time communication with stakeholderswithin DPH and with external partners during events. 